\title{
Role of Intravascular Imaging in Primary $\mathrm{PCl}$
}

\author{
William K. T. Hau and Bryan P. Y. Yan
}

\subsection{Introduction}

Acute ST-segment elevation myocardial infarction (STEMI) usually results from acute thrombotic occlusion of a major epicardial coronary artery. Primary percutaneous coronary intervention (PPCI) is the reperfusion strategy of choice if it can be done in a timely manner, its aim being to rapidly achieve complete myocardial reperfusion. Overwhelming evidence has shown that prompt reperfusion reduces infarct size, preserves left ventricular function, and improves survival [1, 2].

Studies have demonstrated the benefits of drug-eluting stents (DESs) in patients with acute myocardial infarction (MI) undergoing primary stent implantation. The HORIZONS-AMI (Harmonizing Outcomes with Revascularization and Stents in Acute Myocardial Infarction) trial demonstrated that the use of DESs in patients with STEMI is safe and effective compared with bare metal stents (BMSs) at 1-year follow-up. The use of DESs definitively reduces the need for repeat revascularization without additional risk of death or MI [3]. Besides, a meta-analysis of 13 randomized trials, which compared the outcomes of DES and BMS in 7352 randomized patients, suggested that DES use significantly decreases restenosis compared with BMS use in patients with STEMI. DES use yields a relative and absolute reduction in target vessel revascularization (TVR) of 56\% and $-7 \%$, respectively $(p<0.001)$. The meta-analysis also suggested that this benefit does not come at the expense of stent thrombosis, reinfarction, or increased death within 2 years of the PCI [4].

Even though DES use has proven to be safe in the setting of primary PCI, stent thrombosis remains a serious complication associated with increased morbidity and mortality $[5,6]$. Thus, careful attention must be paid to ensure complete stent

B. P. Y. Yan · W. K. T. Hau ( $ه)$

Division of Cardiology, Department of Medicine and Therapeutics, The Chinese University of Hong Kong, Prince of Wales Hospital, Hong Kong, SAR, China

e-mail:william.hau@cuhk.edu.hk 
coverage of the culprit lesion in terms of diameter and stenosis. In STEMI patients, rapid reperfusion is the most crucial issue, so primary PCI is always targeted on the angiographically identified culprit lesion. However, the actual culprit lesion may not be lumen-compromising and could be located proximally or distally to the angiographic target lesion. As a result, the risk of incomplete lesion coverage could be high when the primary PCI is guided solely by angiography. Furthermore, stent implantation must be optimized, as incomplete apposition and/or edge dissection may result in in-stent restenosis or thrombosis. Thus, invasive coronary imaging (Fig. 14.1) using intravascular ultrasound (IVUS) or optical coherence tomography (OCT) is useful for guiding the PCI procedure in the primary setting by locating the true culprit lesion and may lead to better stent coverage of the lesion. Besides, invasive imaging also helps to resolve diagnostic uncertainty and to identify the mechanism underlying the acute event. Thin-cap

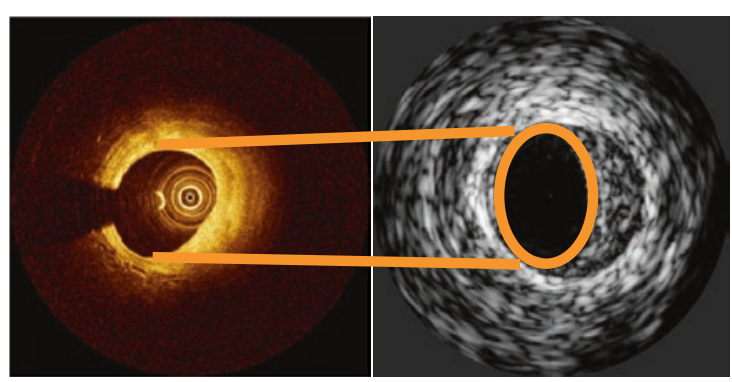

\begin{tabular}{|l|c|c|}
\hline & OCT & IVUS \\
\hline Resolution & $15 \mu \mathrm{m}$ & $150 \mu \mathrm{m}$ \\
\hline Penetration & $2 \mathrm{~mm}$ & $10 \mathrm{~mm}$ \\
\hline Field of View & $10 \mathrm{~mm}$ & $10 \mathrm{~mm}$ \\
\hline Frame Rate & $100 \mathrm{FPS}$ & $15-30 \mathrm{FPS}$ \\
\hline Pullback Speed & $75 \mathrm{~mm} / \mathrm{sec}$ & $0.5 / 1.0 \mathrm{~mm} / \mathrm{sec}$ \\
\hline Catheter Size & Sub 3F & $3.2 \mathrm{~F}$ \\
\hline
\end{tabular}

Fig. 14.1 A comparison table of OCT and IVUS 


\section{Comparing OCT to IVUS}

OCT can visualize $1-2 \mathrm{~mm}$ for seeing the lumen and structures near the lumen

IVUS can penetrate deep into tissue allowing visualization of lumen, stents, entire plaque and vessel wall

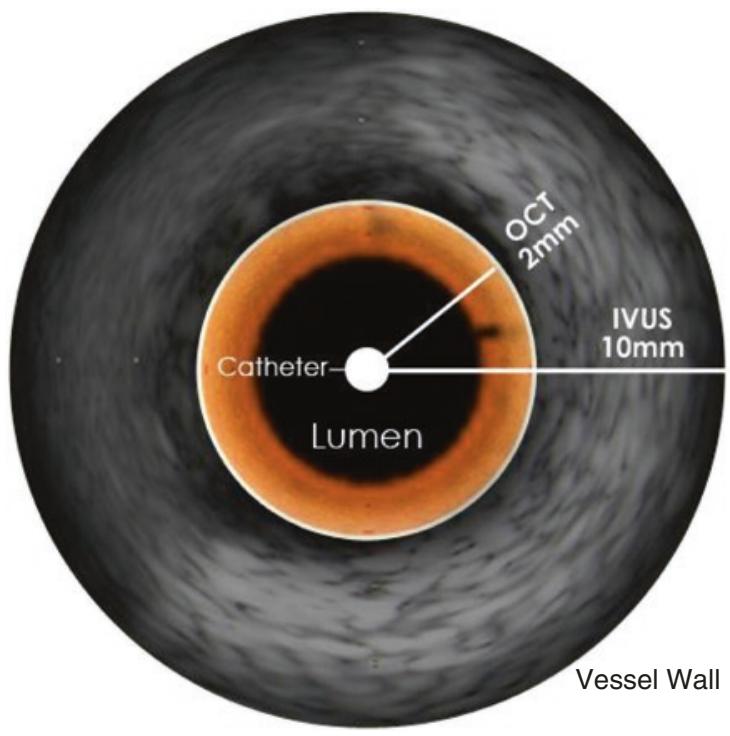

Fig. 14.2 IVUS can penetrate deep into tissue allowing visualization of the lumen, entire plaque, and vessel wall. However, the high resolution of OCT provides superior delineation of the superficial structures

fibroatheroma (TCFA) is the most common type of vulnerable plaque and is defined as a lesion with a fibrous cap thickness $\leq 65 \mu \mathrm{m}$ containing a large lipid pool with a necrotic core and infiltrated by macrophages. It is believed to be the precursor of thrombotic plaque rupture [7]. Autopsy studies have shown that TCFA is more commonly found in coronary segments with positive remodeling [8], and due to its better penetration power than OCT (Fig. 14.2), IVUS is very useful in identifying coronary vessels with positive remodeling [9]. However, the high resolution of OCT is able to provide accurate quantification of fibrous cap thicknesses $\leq 65 \mu \mathrm{m}$ (Fig. 14.3). Other than coronary plaque rupture, plaque erosion and calcified nodules are frequent pathophysiological mechanisms responsible for acute coronary syndrome (ACS) [7], and OCT enables differentiation of all three features in vivo [10]. Other rare causes of acute coronary symptoms such as spontaneous coronary artery dissection [11], myocardial bridging [12], or mechanical problems due to stent underexpansion [13] and/or stent fracture [14] can also be identified by invasive imaging. However, the impact of intravascular imaging in terms of clinical outcomes in the setting of acute MI remains a matter of controversy. 
Fig. 14.3 Thin-cap fibroatheroma (TCFA) is the most common type of vulnerable plaque and is defined as a lesion with a fibrous cap thickness $\leq 65 \mu \mathrm{m}$ containing a large lipid pool with a necrotic core and infiltrated by macrophages. The high resolution of OCT is able to provide accurate quantification of fibrous cap thicknesses $\leq 65 \mu \mathrm{m}$

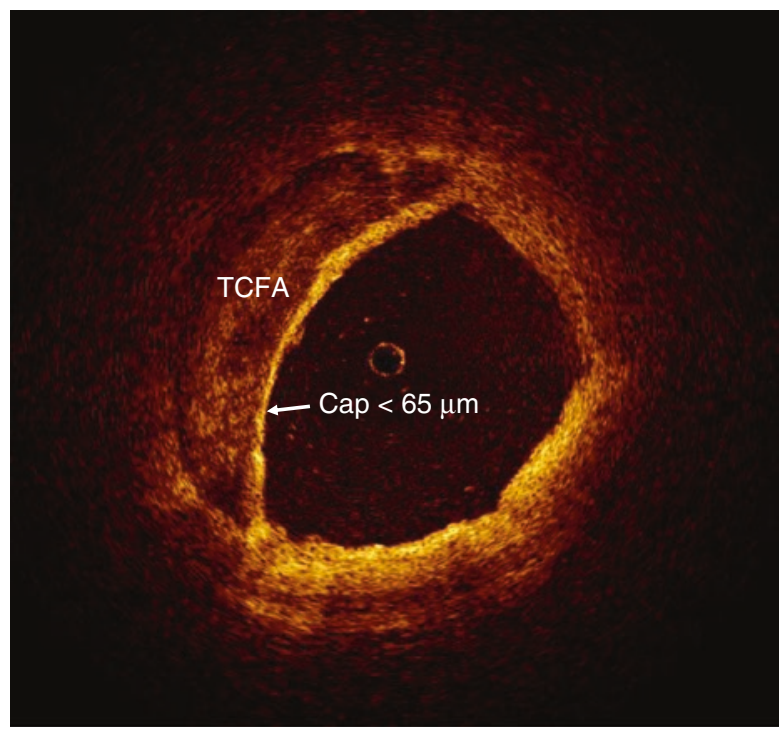

\subsection{Role of Invasive Imaging in the Management of STEMI and Non-STEMI}

\subsubsection{Intravascular Ultrasound}

IVUS uses reflected high-frequency (10-60 MHz) sound waves to visualize the arterial wall in a two-dimensional tomographic format. IVUS permits not only a greater understanding of the characteristics of the coronary plaque and its response to interventional coronary procedures but also allows more precise quantification of the coronary luminal dimensions and atherosclerotic plaque burden [15-17]. In the setting of elective PCI, randomized trials and meta-analyses indicate that IVUSguided PCI is associated with a lower incidence of TVR and fewer major adverse cardiac events (MACEs), MI, and stent thromboses than angiography-guided PCI $[18,19]$. IVUS provides useful information about the morphology of the coronary lesion, as well as helping stent size selection, optimization of stent expansion, and detection of incomplete apposition and/or edge dissection, resulting in reduced restenosis or stent thrombosis. IVUS- measured minimal stent cross-sectional area (CSA) is the best IVUS predictor of DES failure. The results from the IVUS substudy of the HORIZONS-AMI trial showed that mechanical problems such as a smaller final stent CSA $\left(<5 \mathrm{~mm}^{2}\right)$ and inflow/outflow disease (residual stenosis or dissection) but not acute mal-apposition were associated with early stent thrombosis after intervention for acute MI. The minimal stent CSA measured by IVUS and the degree of stent expansion were significantly smaller in patients with early stent thrombosis than in the control group. The finding that the minimal stent CSA was significantly smaller in acute MI patients with early stent thrombosis could be either because of tissue protrusion (plaque and/or thrombus) or stent underexpansion or 
both, as the culprit lesions in acute MI patients are presumed to be thrombuscontaining, and thus tissue protrusion into the lumen through stent struts is common [20]. Besides, another IVUS sub-study of the HORIZONS-AMI trial also suggested that the final post-procedure minimal stent CSA in patients with STEMI after primary stent implantation was the only independent IVUS predictor of angiographic binary restenosis, similar to patients with stable coronary disease as previously reported. Angiographic restenosis rates were $26.7 \%$ in lesions with a CSA $<4 \mathrm{~mm}^{2}$, $22.2 \%$ in lesions with a CSA $<5 \mathrm{~mm}^{2}$, and $20.5 \%$ in lesions with a CSA $<6 \mathrm{~mm}^{2}$ [21]. Therefore, a well-expanded stent with a final minimal stent CSA $\geq 5 \mathrm{~mm}^{2}$ by IVUS is needed in patients undergoing primary PCI to prevent stent thrombosis as well as restenosis at follow-up.

However, it is still controversial whether routine IVUS guidance improves outcomes in patients receiving primary PCI treatment. The Assessment of Dual Antiplatelet Therapy with Drug-Eluting Stents (ADAPT-DES) trial reported that IVUS-guided PCI with DESs was associated with significantly lower rates of stent thrombosis, MI, and MACEs. In this particular trial, 813 STEMI patients were enrolled, and it was found that IVUS use was associated with substantially improved outcomes in these patients [22]. Besides, studies by Singh et al. have shown that the use of IVUS in patients with acute MI was associated with reduced in-hospital mortality but at the expense of increased cost of care and vascular complications, compared with angiography-guided PCI [23]. On the contrary, a few other studies did not support the routine use of IVUS for patients who present with an acute MI and undergoing primary PCI. A study by Maluenda et al. showed no clear clinical benefit of routine use of IVUS-guided stenting in 905 patients with acute MI undergoing primary PCI. The overall rates of the composite primary outcome were similar, as were the rates of stent thrombosis in the IVUSguided and the non-IVUS-guided groups. However, the number of treated lesions and the number of stents used were higher in the IVUS-guided group, with a longer procedural time as well [24]. Consistently, two studies from Korea reported similar findings; IVUS guidance during primary PCI did not provide a better clinical outcome and prevent stent thrombotic events. Although the stent size and the final minimal stented CSA were larger in the IVUS-guided group, the use of IVUS was also associated with a longer stent length and higher number of stents used $[25,26]$. Traditionally, longer and more stents used are associated with much worse clinical outcomes and thus may minimize the potential benefits of IVUS use in acute MI patients undergoing primary PCI. Supporting this notion, a recent study from Japan, the CREDO (Coronary Revascularization Demonstrating Outcome)-Kyoto AMI registry sub-analysis, evaluated the outcomes of 932 patients undergoing IVUS-guided PCI among the 3028 STEMI patients enrolled in the registry and also found no apparent benefit of IVUS for reducing TVR and stent thrombosis as well as mortality in STEMI patients undergoing primary PCI [27]. Despite the fact that, in the setting of elective PCI, IVUS use has been demonstrated to reduce the MACE rate and improve clinical outcomes, in the setting of primary PCI, it seems that IVUS use can be a two-edged sword; it may help in stent implantation guidance-and in terms of proper stent size selection and 


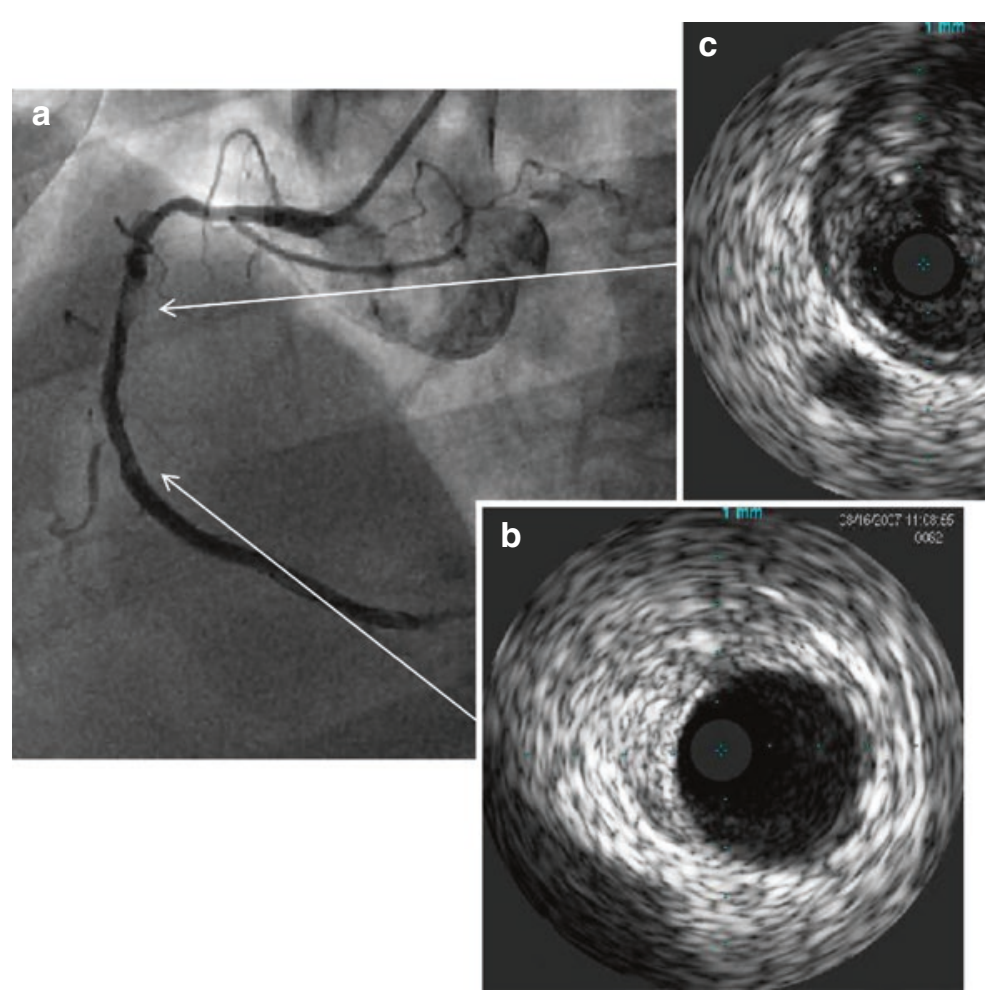

Fig. 14.4 (a) Coronary angiography suggestive of thrombus formation at the mid-right coronary artery. (b) IVUS image of the normal reference distal to the culprit site. (c) IVUS image at the culprit site with positive remodeling contains a large thrombus $(\mathrm{F})$ burden

postimplantation stent optimization, it may result in reduction of restenosis or stent thrombosis. On the other hand though, it may also be associated with a higher rate of coronary complications, and this could be because of poor IVUS interpretation due to a large thrombus burden in the culprit lesions of STEMI patients (Fig. 14.4), making it rather difficult to determine proper stent sizing and optimization of stent deployment, resulting in under- or oversize stent selection, and this may lead to disastrous consequences like perforation and stent thrombosis. In addition, the underlying pathological process in acute settings is very different from that in elective settings. The culprit lesions responsible for causing the acute events are very soft with less calcification and naturally contain a large thrombus burden; thus post-dilatation to optimize stent deployment with highpressure ballooning in acute MI patients may potentially cause thrombus protrusion or tissue prolapse through the stent strut and increase the likelihood of distal embolization. Besides, acute MI patients might have a higher chance of lateacquired stent mal-apposition due to the thrombus resolving over time, and that may lead to thrombotic events as well. It has been shown on IVUS that at the time of acute MI, IVUS use is not able to predict the occurrence of late-acquired 
incomplete stent apposition, as this could result from either thrombus dissolution and/or positive vessel wall remodeling [28]. Thus, it is possible that IVUS use in the setting of primary PCI may increase the overall procedural time and cost without providing additional clinical outcome benefits.

\subsubsection{Optical Coherence Tomography}

OCT generates real-time high-resolution cross-sectional images of the vascular wall from backscattered reflections of infrared light. The greatest advantage of this infrared light-based imaging technology is its significantly higher resolution (tenfold higher) than that of the sound-based technology, but at the cost of having to inject contrast medium to flush blood from the vessel, as the OCT signal is attenuated by the presence of red blood cells. Current commercially available intravascular OCT technologies have an axial resolution of 10-20 $\mu \mathrm{m}$ and a lateral resolution of 20-25 $\mu \mathrm{m}$. The higher resolution of OCT provides superior delineation of the superficial structures and is able to visualize calcium (Fig. 14.5) without acoustic shadowing [29], as seen with IVUS. However, signal penetration through the diseased vessel is more limited due to its lower penetration power, making it difficult to quantify the plaque burden or to investigate large vessels. Besides, since OCT requires the injection of a contrast medium during image acquisition in order to create a blood-free environment, OCT cannot be performed in scenarios such as totally occluded vessels or coronary arteries with massive dissection. The results from the CLI-OPCI (Centro per la Lotta contro l'Infarto-Optimisation of Percutaneous Coronary Intervention) registry suggested that the use of OCT in patients undergoing PCI could improve clinical outcomes. A significant reduction in the primary end
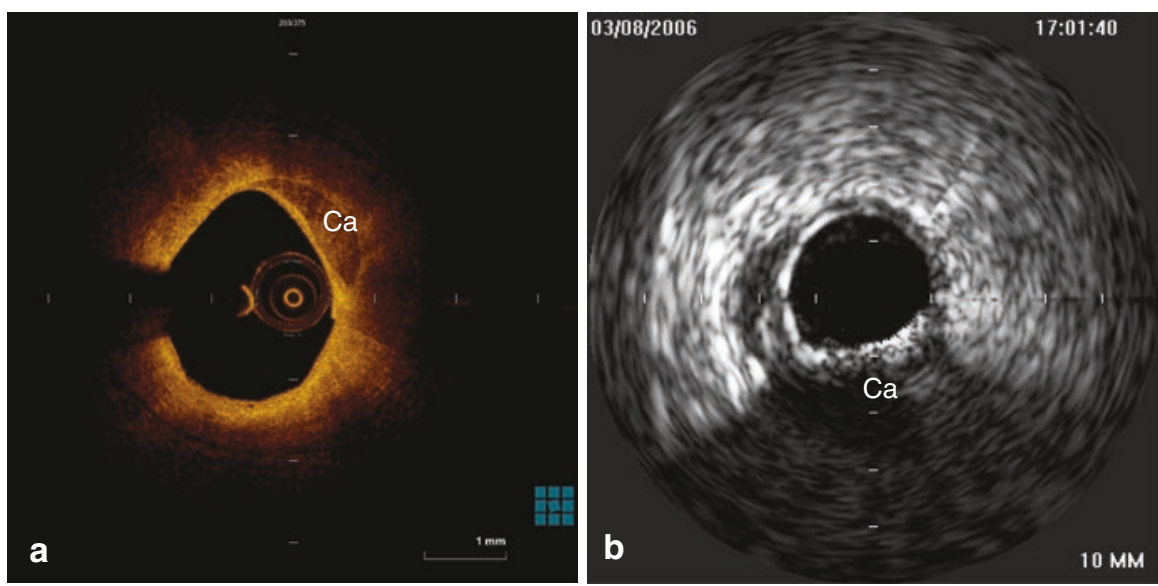

Fig. 14.5 (a) The higher resolution of OCT is able to visualize calcium (Ca) without acoustic shadowing. (b) The IVUS image shows superficial calcium (Ca) from 4 o'clock to 8 o'clock with acoustic shadowing 
point of cardiac death or MI was found in patients undergoing OCT-guided PCI compared with patients treated with angiographic guidance alone [30]. Suboptimal stent deployment was associated with an increased risk of MACEs during followup. More recently, a randomized controlled trial reported that OCT-guided PCI procedures using a specific reference segment external elastic lamina-based stent optimization strategy resulted in clinical outcomes equivalent to those with IVUSguided PCI [31]. Due to the ultra-high resolution, OCT is more sensitive in detecting mal-apposition, tissue prolapse, small edge dissection, and intra-stent thrombus than IVUS. However, studies have also shown that small dissections and tissue protrusion detected by OCT tend to resolve over a 6-month follow-up period [32], so all these small details detected by OCT may be clinically irrelevant and could potentially lead to unnecessary interventions and might eventually cause more complications.

Most acute coronary events, including MI, occur in relation to subsequent coronary thrombus formation. Due to its high resolution, OCT can identify a thrombus better than IVUS and is able to discriminate two types of thrombi (Fig. 14.6): red thrombus (red blood cell-rich), which has high backscatter and high attenuation, and white thrombus (platelet-rich) characterized by signal-rich low backscatter and low attenuation [33]. However, the impact of characterization of these two types of thrombi in clinical decision-making is still unclear, and further studies are needed. Furthermore, in the ACS setting, OCT has been shown to identify plaque morphologies associated with a worse prognosis. Plaque erosion and plaque rupture (Fig. 14.7) are the two most frequent pathophysiological mechanisms responsible for ACS and contribute to almost $95 \%$ of the acute coronary events; OCT enables the differentiation of these two plaque features in vivo. At OCT, plaque erosion appears as a plaque covered by thrombus (typically a white, platelet-rich thrombus) without signs of fibrous cap disruption. This occurs at sites with impaired endothelium, rich in smooth muscle cells and proteoglycans. In contrast, plaque rupture is identified by the presence of a fibrous cap
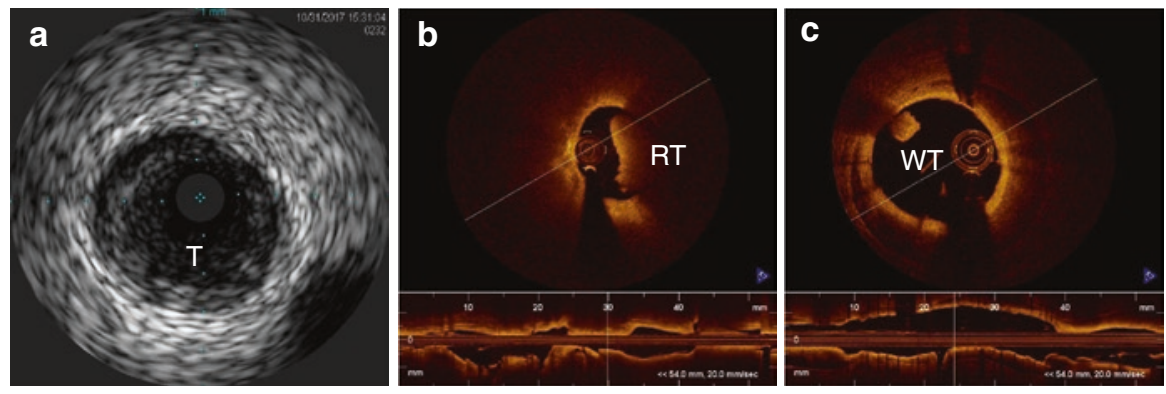

Fig. 14.6 (a) IVUS image of a vessel contains a large thrombus burden. OCT is able to discriminate two types of thrombi: (b) red thrombus, which has high backscatter and high attenuation, and (c) white thrombus characterized by signal-rich low backscatter and low attenuation. $T$ thrombus, $R T$ red thrombus, $W T$ white thrombus 

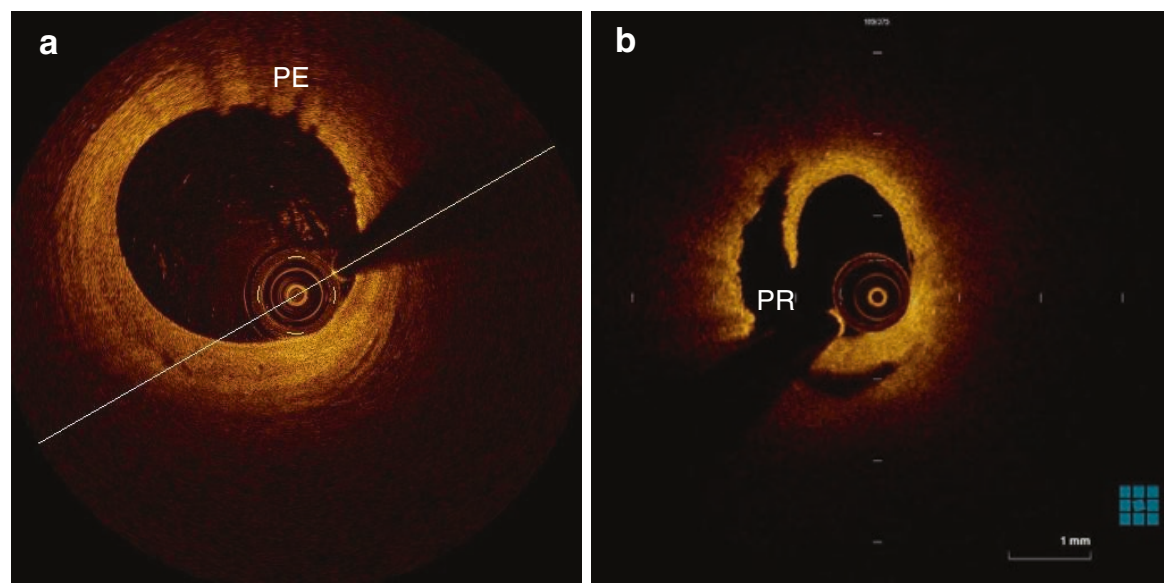

Fig. 14.7 OCT enables the differentiation of plaque erosion (PE) and plaque rupture (PR) in vivo. (a) PE appears as a plaque covered by thrombus (typically a white, platelet-rich thrombus) without signs of fibrous cap disruption. (b) PR is identified by the presence of a fibrous cap discontinuity, creating communication between a cavity within the plaque and the arterial lumen

discontinuity, creating communication between a cavity within the plaque and the arterial lumen. Calcified nodules contribute to $\leq 5 \%$ of acute coronary events, and these are characterized by fibrous cap disruption due to protrusion of calcium [34, 35]. Differentiation of these three plaque features can be useful in the choice of therapeutic strategy. The recent EROSION (Effective Antithrombotic Therapy Without Stenting: Intravascular OCT-Based Management in Plaque Erosion) trial suggested that patients with ACS caused by plaque erosion may be stabilized by antithrombotic therapy alone, without stenting, and thereby potentially have fewer stent-related early and late complications [36].

The high resolution of OCT clearly enables a better understanding of the atherosclerotic plaque and helps to identify the mechanism underlying the acute event. Studies aiming to investigate an association between OCT and improvement in clinical outcomes may further strengthen the evidence in favor of using OCT to guide PCI in ACS patients. The DOCTORS (Does Optical Coherence Tomography Optimize Results of Stenting) trial is the first randomized, prospective, multicenter trial to investigate the use of OCT in optimizing the results of PCI for non-STsegment elevation ACS. The results showed that OCT findings led to a change in procedural strategy in 50\% of the patients in the OCT-guided group, mainly driven by the optimization of stent expansion, and were associated with higher fractional flow reserve values at the end of the procedure than angiography-guided PCI alone. However, this benefit was obtained at the cost of longer fluoroscopy and procedural times, as well as a greater volume of contrast medium and a higher dose of radiation, but without an increase in periprocedural complications, MI, or kidney dysfunction [37]. 


\subsection{Role of Invasive Imaging in the Management of Spontaneous Coronary Artery Dissection}

\subsubsection{Pathogenesis of Spontaneous Coronary Artery Dissection}

The usual pathogenesis of acute MI involves unstable plaque rupture or plaque erosion that is distinct from spontaneous coronary artery dissection (SCAD). SCAD is a rare cause of acute $\mathrm{MI}$, and is due to hemorrhage within the arterial wall, resulting in separation of the intimal-medial layers rather than atherosclerotic plaque rupture or erosion. The presence of true arterial lumen compression by the hematoma in the intimal-medial layers can subsequently result in myocardial ischemia or acute MI. Early recognition and precise diagnosis of SCAD is very important in order to implement the appropriate medical treatment; otherwise it will lead to disastrous consequences. Two potential mechanisms for the cause of SCAD have been proposed. The first is that an intimal tear creates an entry point that promotes intramural bleeding inside the false lumen, leading to separation of the intimal-medial layers. The second is that rupture of the vasa vasorum leads to an intramural hematoma, which increases the pressure and potentially causes an intimal rupture into the true lumen. Thus SCAD caused by the latter mechanism can occur with or without a distinct intimal rupture [38]. SCAD is not uncommon in young females presenting with acute MI in the absence of traditional cardiovascular risk factors [39] and is the most frequent cause of acute MI among pregnant women [40].

The symptoms of SCAD usually do not differ from those of ACS, as SCAD impairs myocardial perfusion by reducing the vessel lumen in a manner very similar to atherosclerotic stenosis, and thus accurate recognition and management of SCAD may improve both short- and long-term outcomes. However, SCAD is mainly caused by spontaneous dissection of the coronary artery wall. As a matter of fact, it is very difficult to correctly diagnose based on angiography alone as angiography is only a two-dimensional luminogram and is not able to visualize the vessel wall, explaining why SCAD is frequently underdiagnosed. The angiographic appearance of SCAD consists of extraluminal contrast staining, spiral dissection, an intraluminal filling defect, and smooth narrowing of varying length and severity which depends on the amount of blood accumulation within the false lumen. Based on the angiographic appearance, Saw proposed a classification of SCAD (Fig. 14.8) to aid its diagnosis [41]. Type 1 angiographic SCAD describes the pathognomonic appearance of arterial wall contrast staining with multiple radiolucent lumens. Type 2 angiographic SCAD describes long diffuse stenosis of varying severity (typically $>20 \mathrm{~mm}$ ), but often subtle, abrupt changes in arterial caliber from the normal diameter to diffuse smooth narrowing, and often extends to the distal ends of the arteries. Type 3 angiographic SCAD describes focal tubular (typically $<20 \mathrm{~mm}$ ) stenosis that mimics atherosclerotic lesions. Invasive coronary imaging techniques like IVUS and OCT that can image the arterial wall improve SCAD diagnosis and are also very useful in guiding coronary intervention when revascularization is needed. However, they are not available in some catheterization laboratories and are also associated with additional risks and cost. Thus, angiography remains the frontline imaging 

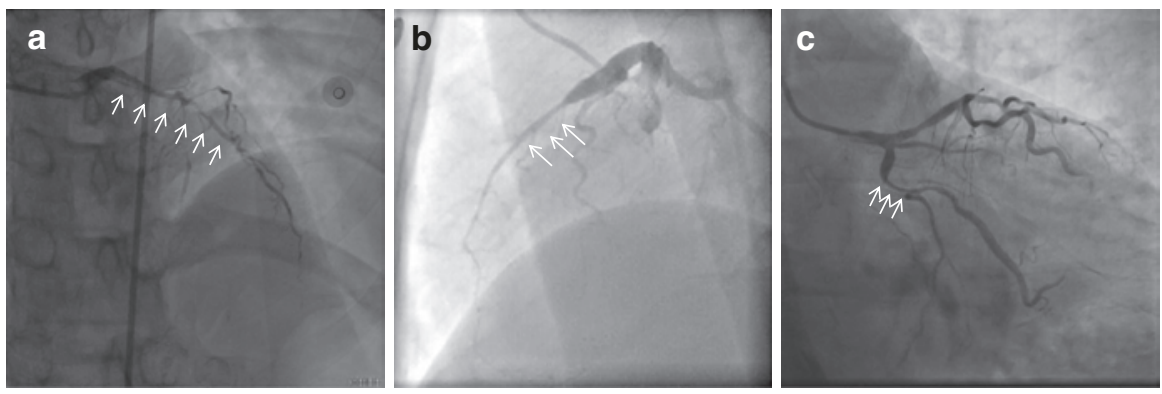

Fig. 14.8 Saw proposed an angiographic classification of SCAD to aid its diagnosis. (a) Type 1 angiographic SCAD describes the pathognomonic appearance of arterial wall contrast staining with multiple radiolucent lumens. (b) Type 2 angiographic SCAD describes long diffuse stenosis of varying severity (typically $>20 \mathrm{~mm}$ ), but often subtle, abrupt changes in arterial caliber from the normal diameter to diffuse smooth narrowing, and often extends to the distal ends of the arteries. (c) Type 3 angiographic SCAD describes focal tubular (typically $<20 \mathrm{~mm}$ ) stenosis that mimics atherosclerotic lesions

technology for the diagnosis of SCAD. However, when it comes to SCAD patient management, coronary revascularization is only indicated for those with ongoing or recurrent ischemia with favorable anatomy but is associated with high complication rates [42]. On the other hand, patients without evidence of ongoing ischemia can be treated medically, and most have an uneventful clinical course [43]. Medical management consists of blood pressure control with dual antiplatelet and beta-blockers.

\subsubsection{Intravascular Ultrasound}

As noted above, in both elective and acute settings, IVUS is very useful in the determination of the severity and characteristics of atherosclerotic plaques and has played a vital role in PCI guidance in terms of vessel sizing and the optimization of stent implantation. In patients suspected of having SCAD, the diagnosis of SCAD requires visualization of the intramural hematoma and separation of the intimal-medial layers creating double true and false lumens. Thus, due to its better tissue penetration power, IVUS allows a more complete and deeper vessel visualization of the extent of the intramural hematoma, as well as the dissected layers, while the true and false lumens can be clearly identified. Also, the distribution of the false lumen and the compromise of the true lumen can also be clearly visualized on IVUS [44]. An intramural hematoma on IVUS appears homogeneous and hyperechoic, with a crescent-shaped area of blood accumulation (Fig. 14.9). However, due to its relatively poor image resolution, IVUS is not able to identify the intimal rupture site. On the other hand, IVUS is able to confirm the guide-wire position within the true lumen (Fig. 14.10), and IVUS findings have also been used to optimize the results of stent implantation in patients with SCAD requiring coronary intervention [45]. 

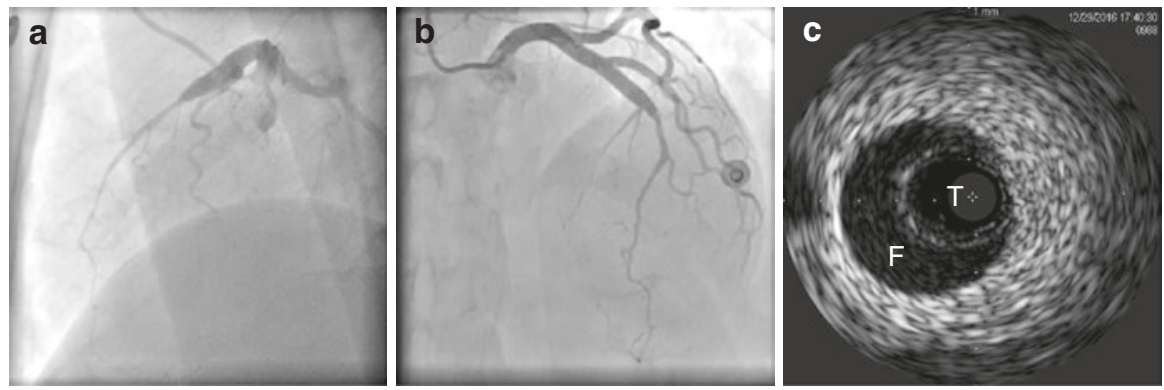

Fig. 14.9 (a) and (b) Coronary angiography suggesting a type 2 SCAD. (c) IVUS revealing the presence of a dissection; both the true $(\mathrm{T})$ and false $(\mathrm{F})$ lumens can be clearly identified

Fig. 14.10 IVUS

confirms the guide-wire $(\mathrm{GW})$ is in the true (T) lumen, not the false $(\mathrm{F})$ lumen

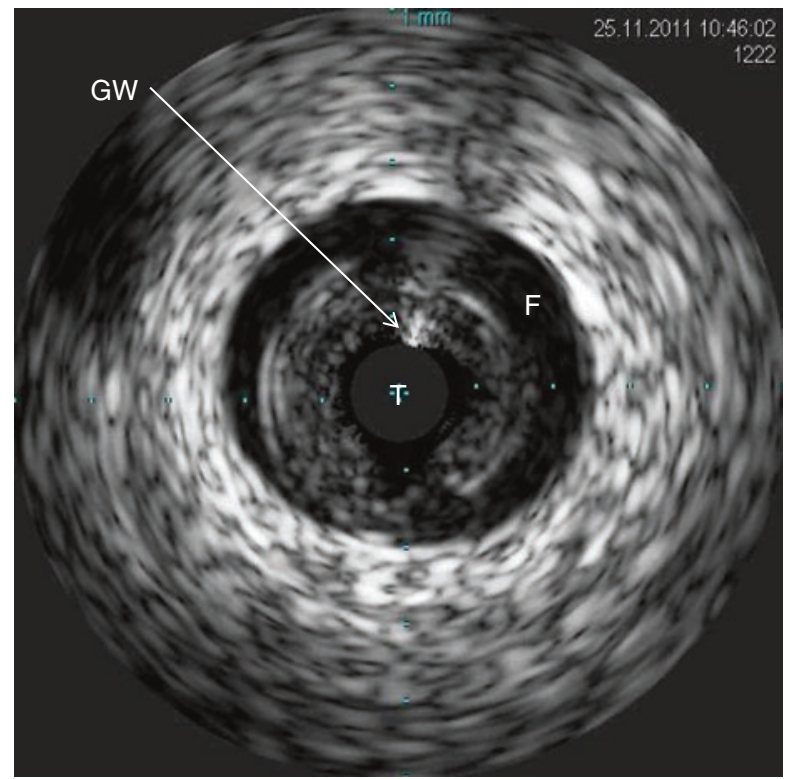

\subsubsection{Optical Coherence Tomography}

Coronary angiography is unable to establish a definitive diagnosis of SCAD. Conversely, OCT allows accurate visualization of the coronary artery wall with unprecedented resolution and thus is very useful to rule out this diagnosis in patients with images mimicking SCAD. OCT provides unique insights by visualizing most of the morphological features of the condition including intimal tears, intraluminal thrombi, double-lumen morphology, and intramural hematoma. However, inadequate optical penetration may impede full visualization of the circumferential extent of an intramural hematoma. Like IVUS, the distribution of the false lumen and the compromise of the true lumen can also be very clearly visualized on OCT. An intramural hematoma, on the other hand, has a dark appearance on OCT (Fig. 14.11). 
In addition, due to its superb near-field resolution compared with IVUS, OCT is superior in identifying the precise location of the intimal rupture tear and readily depicts the length of the dissecting membrane [46]. However, IVUS provides deeper penetration of the vessel wall and is therefore able to fully visualize large hematomas (Fig. 14.12). On the other hand, caution must be exercised when performing OCT imaging on SCAD patients, as OCT requires flush injection of contrast medium to
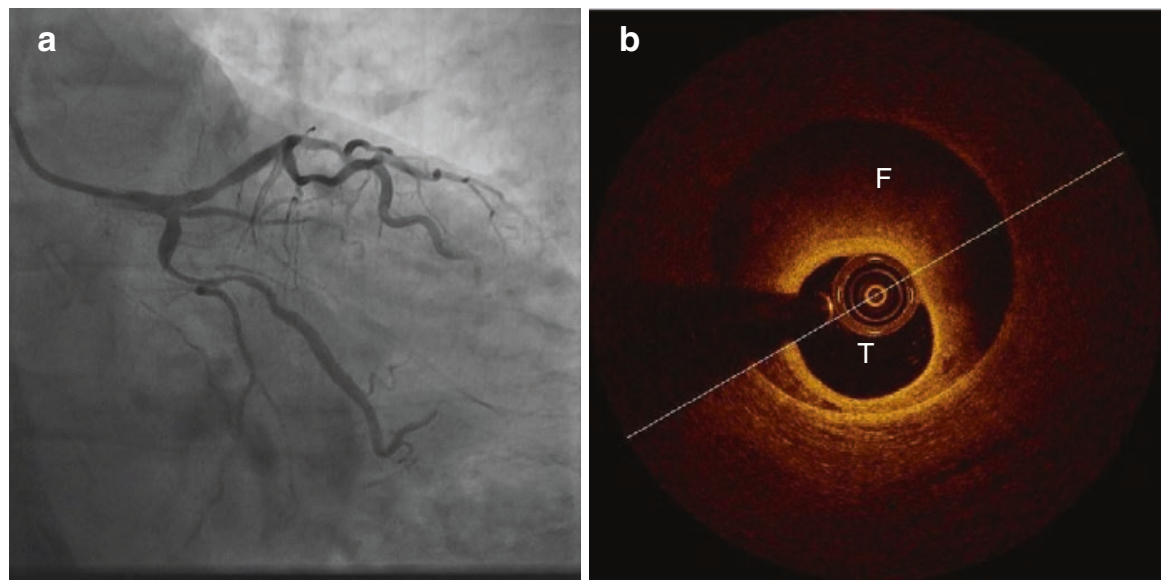

Fig. 14.11 (a) Coronary angiography suggesting a type 3 SCAD. (b) OCT revealing the presence of a dissection; both the true $(\mathrm{T})$ and false $(\mathrm{F})$ lumens can be clearly identified
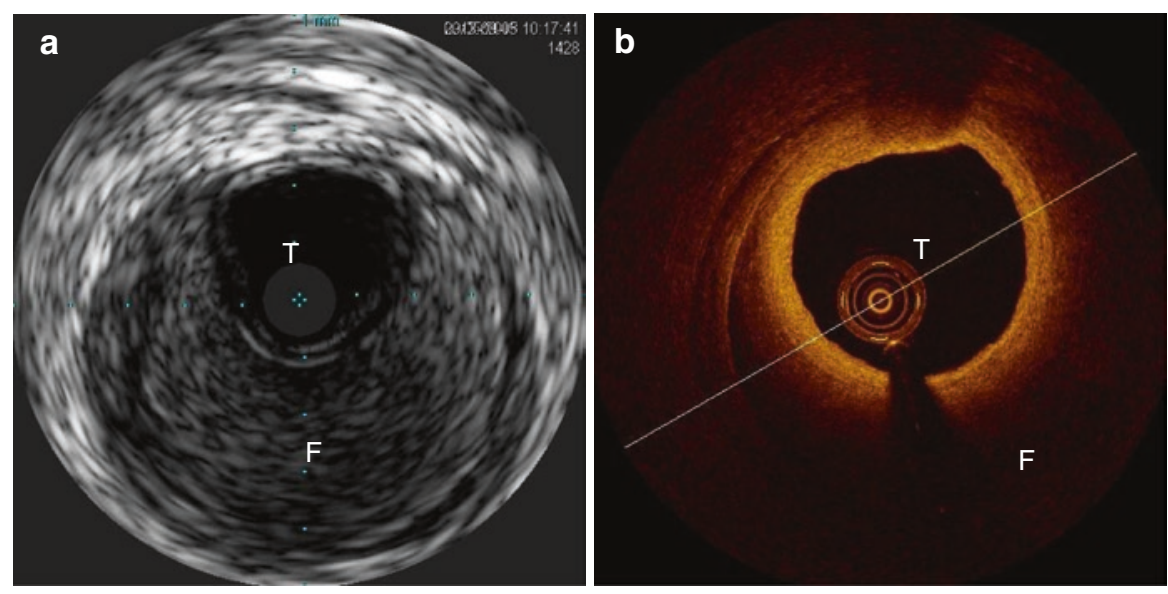

Fig. 14.12 (a) Intramural hematoma on IVUS appears homogeneous and hyperechoic, with a crescent-shaped area of blood accumulation. The distribution of the false lumen (F) and the compromise of the true lumen (T) are clearly visualized. (b) Intramural hematoma has a dark appearance on OCT. The distribution of the false lumen (F) and the compromise of the true lumen (T) can also be very clearly visualized on OCT. However, IVUS provides deeper penetration of the vessel wall and is therefore able to fully visualize large hematomas 
create a blood-free environment during image acquisition in order to obtain good images. This may cause the hematoma to extend distally. Also, abnormalities such as an extramural vessel hematoma or perivascular hematoma can be detected on IVUS, but not on OCT. Finally, for patients undergoing coronary intervention, OCT imaging is also able to help to confirm the guide-wire position within the true lumen, as well as helping in vessel sizing and stent optimization [47].

\subsection{Summary}

Even though angiography is the gold standard to guide procedural decision-making during primary PCI, it has various well-known limitations. Angiography is only a luminology without providing any information on the vessel wall and atherosclerotic plaque characteristics. Besides, it is suboptimal in detecting stent underexpansion, stent edge dissection, plaque protrusion, or thrombi. Invasive imaging techniques like IVUS and OCT provide complementary details to help primary PCI guidance, such as to ensure stent coverage of the culprit lesion and optimization of stent implantation. OCT is superior in the visualization of superficial structures, such as stent edge dissection, intimal tear, tissue protrusion, and intraluminal thrombi, whereas IVUS is better for deep vessel imaging, vessel sizing, and identifying positive remodeling. However, it remains controversial as to whether routine invasive imaging guidance improves outcomes in patients receiving primary PCI.

\section{References}

1. Keeley EC, Boura JA, Grines CL. Primary angioplasty versus intravenous thrombolytic therapy for acute myocardial infarction: a quantitative review of 23 randomized trials. Lancet. 2003;361:13-20.

2. Mauri L, Silbaugh TS, Garg P, et al. Drug-eluting or bare-metal stents for acute myocardial infarction. N Engl J Med. 2008;359:1330-42.

3. Stone GW, Lansky AJ, Pocock SJ, et al. Paclitaxel-eluting stents versus bare-metal stents in acute myocardial infarction. N Engl J Med. 2009;360:1946-59.

4. Brar SS, Leon MB, Stone GW, et al. Use of drug-eluting stents in acute myocardial infarction. A systematic review and meta-analysis. J Am Coll Cardiol. 2009;53:1677-89.

5. Sianos G, Papafaklis MI, Darmen J, et al. Angiographic stent thrombosis after routine use of drug-eluting stents in ST-segment elevation myocardial infarction: the importance of thrombus burden. J Am Coll Cardiol. 2007;50:573-83.

6. Iakovou I, Schmidt T, Bonizzoni E, et al. Incidence, predictors, and outcome of thrombosis after successful implantation of drug-eluting stents. JAMA. 2005;293:2126-30.

7. Virmani R, Kolodgie FD, Burke AP, et al. Lessons from sudden coronary death: a comprehensive morphological classification scheme for atherosclerotic lesions. Arterioscler Thromb Vasc Biol. 2000;20:1262-75.

8. Burke AP, Farb A, Malcolm GT, et al. Coronary risk factors and plaque morphology in men with coronary disease who died suddenly. N Engl J Med. 1997;336:1276-82.

9. Nakamura M, Nishikawa H, Mukai S, et al. Impact of coronary artery remodeling on clinical presentation of coronary artery disease: an intravascular ultrasound study. J Am Coll Cardiol. 2001;37:63-9. 
10. Niccoli G, Montone RA, DiVito L, et al. Plaque rupture ad intact fibrous cap assessed by optical coherence tomography portend different outcomes in patients with acute coronary syndrome. Eur Heart J. 2015;36:1377-84.

11. Tweet MY, Hayes SN, Pitta SR, et al. Clinical features, management, and prognosis of spontaneous coronary artery dissection. Circulation. 2012;126:579-88.

12. Ishikawa Y, Akasaka Y, Akishima-Fukasawa Y, et al. Histopathologic profiles of coronary atherosclerosis by myocardial bridge underlying myocardial infarction. Atherosclerosis. 2013;226:118-23.

13. Fujii K, Carlier SG, Mintz GS, et al. Stent underexpansion and residual reference segment stenosis are related to stent thrombosis after sirolimus-eluting stent implantation. J Am Coll Cardiol. 2005;45:995-8.

14. Makaryus AN, Lefkowitz L, Lee AD. Coronary artery stent fracture. Int J Cardiovasc Imaging. 2007;23:305-9.

15. Mintz GS, Painter JA, Pichard AD, et al. Atherosclerosis in angiographically "normal" coronary artery reference segments: an intravascular ultrasound study with clinical correlations. J Am Coll Cardiol. 1995;25:1479-85.

16. Mintz GS. Intravascular ultrasound and outcome after drug-eluting stent implantation. Coron Artery Dis. 2017;28:346-52.

17. Mintz GS, Nissen SE, Anderson WD, et al. American college of cardiology clinical expert consensus document on standards for acquisition, measurement and reporting of intravascular ultrasound studies (IVUS): a report of the American college of cardiology task force on clinical expert consensus documents. J Am Coll Cardiol. 2001;37:1478-92.

18. Steinvil A, Zhang YJ, Lee SY, et al. Intravascular ultrasound-guided drug eluting stent implantation: an updated meta-analysis of randomized control trials and observational studies. Int J Cardiol. 2016;216:133-9.

19. Bavishi C, Sardar P, Chatterjee $S$, et al. Intravascular ultrasound-guided vs angiography-guided drug-eluting stent implantation in complex coronary lesions: meta-analysis of randomized trials. Am Heart J. 2017;185:26-34.

20. Choi SY, Witzenbichler B, Maehara A, et al. Intravascular ultrasound findings of early stent thrombosis after primary percutaneous intervention in acute myocardial infarction. A harmonizing outcomes with revascularization and stents in acute myocardial infarction (HORIZONSAMI) substudy. Circ Cardiovasc Interv. 2001;4:239-47.

21. Choi SY, Maehara A, Cristea E, et al. Usefulness of minimum stent cross sectional area as a predictor of angiographic restenosis after primary percutaneous coronary intervention in acute myocardial infarction (from the HORIZONS-AMI trial IVUS substudy). Am J Cardiol. 2012;109:455-60.

22. Witzenbichler B, Maehara A, Weisz G, et al. Relationship between intravascular ultrasound guidance and clinical outcomes after drug-eluting stents: the assessment of dual antiplatelet therapy with drug-eluting stents (ADAPT-DES) study. Circulation. 2014;129:463-70.

23. Singh V, Badheka AO, Arora S, et al. Comparison of inhospital mortality, length of hospitalization, costs, and vascular complications of percutaneous coronary interventions guided by ultrasound versus angiography. Am J Cardiol. 2015;115:1357-66.

24. Maluenda G, Lemesle G, Ben-Dor I, et al. Impact of intravascular ultrasound guidance in patients with acute myocardial infarction undergoing percutaneous coronary intervention. Catheter Cardiovasc Interv. 2010;75:86-92.

25. Ahmed K, Jeong MH, Chakraborty R, et al. Role of intravascular ultrasound in patients with acute myocardial infarction undergoing percutaneous coronary intervention. Am J Cardiol. 2011;108:8-14.

26. Youn YJ, Yoon JH, Lee JW, et al. Intravascular ultrasound-guided primary percutaneous coronary intervention with drug-eluting stent implantation in patients with ST-segment elevation myocardial infarction. Clin Cardiol. 2011;34:706-13.

27. Nakatsuma K, Shiomi H, Morimoto T, et al. Intravascular ultrasound guidance vs. angiographic guidance in primary percutaneous coronary intervention for ST-segment elevation 
myocardial infarction - long term clinical outcomes from the CREDO-Kyoto AMI registry. Circ J. 2006;80:477-84.

28. Cheneau E, Leborgne L, Mintz GS, et al. Predictors of subacute stent thrombosis results of a systematic intravascular ultrasound study. Circulation. 2003;108:43-7.

29. Kume T, Okura H, Kawamoto T, et al. Assessment of the coronary calcification by optical coherence tomography. EuroIntervention. 2011;6:768-72.

30. Prati F, Di Vito L, Biondi-Zoccai G, et al. Angiography alone versus angiography plus optical coherence tomography to guide decision-making during percutaneous coronary intervention: the Centro per la Lotta contro I'Infarto-Optimisation of Percutaneous Coronary Intervention (CLI-OPCI) study. EuroIntervention. 2012;8:823-9.

31. Ali ZA, Maehara A, Genereux P, et al. Optical coherence tomography compared with intravascular ultrasound with angiography to guide coronary stent implantation (ILUMIEN III: OPTIMIZE PCI): a randomized controlled trial. Lancet. 2016;388:2618-28.

32. Kawamori H, Shite J, Shinke T, et al. Natural consequence of post-intervention stent malapposition, thrombus, tissue prolapse, and dissection assessed by optical coherence tomography at mid-term follow-up. Eur Heart J Cardiovasc Imaging. 2013;14:865-75.

33. Kume T, Akasaka T, Kawamoto T, et al. Assessment of coronary arterial thrombus by optical coherence tomography. Am J Cardiol. 2006;97:1713-7.

34. Kubo T, Imanishi T, Takarada S, et al. Assessment of culprit lesion morphology in acute myocardial infarction: ability of optical coherence tomography compared with intravascular ultrasound and coronary angioscopy. J Am Coll Cardiol. 2007;50:933-9.

35. Jia H, Abtahian F, Aguirre AD, et al. In vivo diagnosis of plaque erosion and calcified nodule in patients with acute coronary syndrome by intravascular optical coherence tomography. J Am Coll Cardiol. 2013;62:1748-58.

36. Jia H, Dai J, Hou J, et al. Effective anti-thrombotic therapy without stenting: intravascular optical coherence tomography-based management in plaque erosion (the EROSION study). Eur Heart J. 2017;38:792-800.

37. Meneveau N, Souteyrand G, Motreff P, et al. Optical coherence tomography to optimize results of percutaneous coronary intervention in patients with non-ST-elevation acute coronary syndrome - results of multicenter randomized DOCTORS study (does optical coherence tomography optimize results of stenting). Circulation. 2016;134:906-17.

38. Maehara A, Mintz GS, Castagna MT, et al. Intravascular ultrasound assessment of spontaneous coronary artery dissection. Am J Cardiol. 2002;89:466-8.

39. Tweet MS, Best P, Hayes SN. Unique presentations and etiologies of myocardial infarction in women. Curr Treat Options Cardiovasc Med. 2017;19:66.

40. Tweet MS, Hayes SN, Codsi E, et al. Spontaneous coronary artery dissection associated with pregnancy. J Am Coll Cardiol. 2017;70:426-35.

41. Saw J. Coronary angiogram classification of spontaneous coronary artery dissection. Catheter Cardiovasc Interv. 2014;84:1115-22.

42. Tweet MS, Eleid MF, Best PJM, et al. Spontaneous coronary artery dissection revascularization versus conservative therapy. Circ Cardiovasc Interv. 2014;7:777-86.

43. Alfonso F, Paulo M, Lennie V, et al. Spontaneous coronary artery dissection: long-term followup of a large series of patients prospectively managed with a "conservative" therapeutic strategy. J Am Coll Cardiol Intv. 2012;5:1061-70.

44. Maehara A, Mintz GS, Bui AB, et al. Incidence, morphology, angiographic findings, and outcomes of intramural hematomas after percutaneous coronary interventions: an intravascular ultrasound study. Circulation. 2002;105:2037-3042.

45. Arnold JR, West NEJ, Gaal WJV, et al. The role of intravascular ultrasound in the management of spontaneous coronary artery dissection. Cardiovasc Ultrasound. 2008;6:24.

46. Alfonso F, Paulo M, Gonzalo N, et al. Diagnosis of spontaneous coronary artery dissection by optical coherence tomography. J Am Coll Cardiol. 2012;59:1073-9.

47. Nakagawa M, Shite J, Shinke T, et al. Ability of optical coherence tomography to visualize the entry port of spontaneous coronary artery dissection. Circ J. 2011;75:2505-7. 
Open Access This chapter is licensed under the terms of the Creative Commons Attribution 4.0 International License (http://creativecommons.org/licenses/by/4.0/), which permits use, sharing, adaptation, distribution and reproduction in any medium or format, as long as you give appropriate credit to the original author(s) and the source, provide a link to the Creative Commons license and indicate if changes were made.

The images or other third party material in this chapter are included in the chapter's Creative Commons license, unless indicated otherwise in a credit line to the material. If material is not included in the chapter's Creative Commons license and your intended use is not permitted by statutory regulation or exceeds the permitted use, you will need to obtain permission directly from the copyright holder.

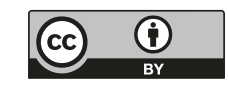

